\title{
Incidence of total hip or knee replacement due to osteoarthritis in relation to thyroid function: a prospective cohort study (The Nord-Trøndelag Health Study)
}

Alf Inge Hellevik ${ }^{1,2^{*}}$, Marianne Bakke Johnsen ${ }^{3,6}$, Arnulf Langhammer ${ }^{1}$, Anne Marie Fenstad ${ }^{4}$, Ove Furnes ${ }^{4,5}$, Kjersti Storheim ${ }^{3,6}$, John Anker Zwart ${ }^{3,6}$, Gunnar Flugsrud² and Lars Nordsletten 2,6

\begin{abstract}
Background: To study whether thyroid function was associated with risk of hip or knee replacement due to primary osteoarthritis.

Methods: In a prospective cohort study, data from the second and third survey of the Nord-Trøndelag Health Study were linked to the Norwegian Arthroplasty Register in order to identify total hip or knee replacement as a result of primary osteoarthritis.

Results: Among 37891 participants without previously known thyroid disease we recorded 978 total hip replacements (THRs) and 538 total knee replacements (TKRs) during a median follow-up time of 15.7 years. The analyses were adjusted for sex, age, BMI (body mass index), smoking, physical activity and diabetes. We did not find any association between TSH (thyroid stimulating hormone) and THR or TKR due to osteoarthritis. Neither were changes in TSH over time, or overt hypo- or hyperthyroidism, associated with incidence of THR or TKR.
\end{abstract}

Conclusion: No association was found between thyroid function and hip or knee joint replacement due to osteoarthritis.

Keywords: Thyroid function, Thyroid stimulating hormone, Osteoarthritis, Hip joint replacement, Knee joint replacement

\section{Background}

Osteoarthritis in the hip and knee is a major health problem and leads to significant morbidity [1]. Several drugs have been evaluated, but so far only exercise has been found to effectively prevent or delay the onset of osteoarthritis $[2,3]$. This finding emphasizes the importance of identifying modifiable risk factors. Thyroid hormones play a role in the remodelling and maintenance of bone, and recent studies also indicate the potential importance of thyroid hormones in joints and articular cartilage [4]. Genetic studies have suggested

\footnotetext{
* Correspondence: alf.hellevik@ntnu.no

'The HUNT Research Centre, Department of Public Health and Nursing, Faculty of Medicine and Health Sciences, Norwegian University of Science and Technology (NTNU), Levanger, Norway

${ }^{2}$ Division of Orthopaedic Surgery, Oslo University Hospital, Oslo, Norway

Full list of author information is available at the end of the article
}

that deiodinase-regulated local availability of the active thyroid hormone triiodothyronine (T3) plays an important role in cartilage maintenance and repair [5]. Further data have indicated that increased intracellular T3 availability increases the risk of osteoarthritis, leading to the hypothesis that reduced tissue T3 availability protects joints from development of osteoarthritis [6]. A phase III clinical trial investigating the use of eprotirome, a thyroid receptor $\beta$-agonist, for treatment of hypercholesterolemia [7], was terminated due to indications of dose related articular cartilage damage in dogs which had been treated with eprotirome for 12 months [8]. This was surprising, as eprotirome is a liver-specific thyroid receptor $\beta$-agonist, but it indicates that thyroid hormones influence cartilage [9] and could play a role in the pathogenesis of osteoarthritis. 
No prospective population studies have investigated the association between thyroid function and osteoarthritis. An older cross-sectional study did not find any association between radiological knee osteoarthritis and thyroid status measured by thyroid stimulating hormone (TSH) [10]. In this prospective cohort study of 37891 individuals without previously known thyroid disease, the aim was to assess whether thyroid function was associated with subsequent risk of hip or knee replacement due to primary osteoarthritis.

\section{Methods}

In the Nord-Trøndelag Health Study (HUNT) all inhabitants of Nord-Trøndelag county $\geq 20$ years of age were invited to participate in three surveys: HUNT1 (1984-1986), HUNT2 (1995-1997) and HUNT3 (2006-2008) [11]. This study only included data from the HUNT2 and HUNT3 surveys, as the HUNT1 study did not collect blood samples. HUNT2 had 65 237 participants (69.5\% of those invited), and HUNT3 had 50807 participants (54.1\% of those invited) [12].

In HUNT2, TSH was measured in 35269 persons; in all women over 40 years old, in a random $50 \%$ sample of men over 40 years old and in a random $5 \%$ sample of participants aged 20-40 years. In HUNT3, TSH was measured in all 49179 participants. We included 35269 participants from HUNT2 and 13132 new participants with TSH measurements from HUNT3. In persons that participated in both HUNT2 and HUNT3, baseline measurements from HUNT2 were used in the main analyses. Among these 48401 individuals, 10510 were excluded from analysis (Fig. 1). The exclusion criteria included self-reported thyroid disease (hypothyroidism, hyperthyroidism, goitre, other thyroid disease, use of levothyroxine, carbimazole, previous thyroid surgery or radioiodine therapy) $(n=3895)$, missing information on BMI $(n=364)$, missing information on smoking $(n=962)$, previous THR or TKR $(n=644)$, missing date of operation $(n=99)$, emigration during baseline measurements period $(n=1)$ or self-reported osteoarthritis at baseline $(n=4545)$. Thus, a total of 37891 people (22 714 women and 15177 men) were eligible for follow-up in this study. Each participant contributed person-years from baseline (either between August 1995 and June 1997 or between October 2006 and June 2008) until a THR or TKR due to osteoarthritis, THR or TKR due to other causes, migration, death or end of follow-up (December 31, 2013), whichever occurred first.

\section{Measurements}

The participants filled out a self-administered questionnaire, including history of thyroid disease [13]. The survey also included measurements of height and weight by trained personnel. Weight was measured while the participants were wearing light clothing without shoes.

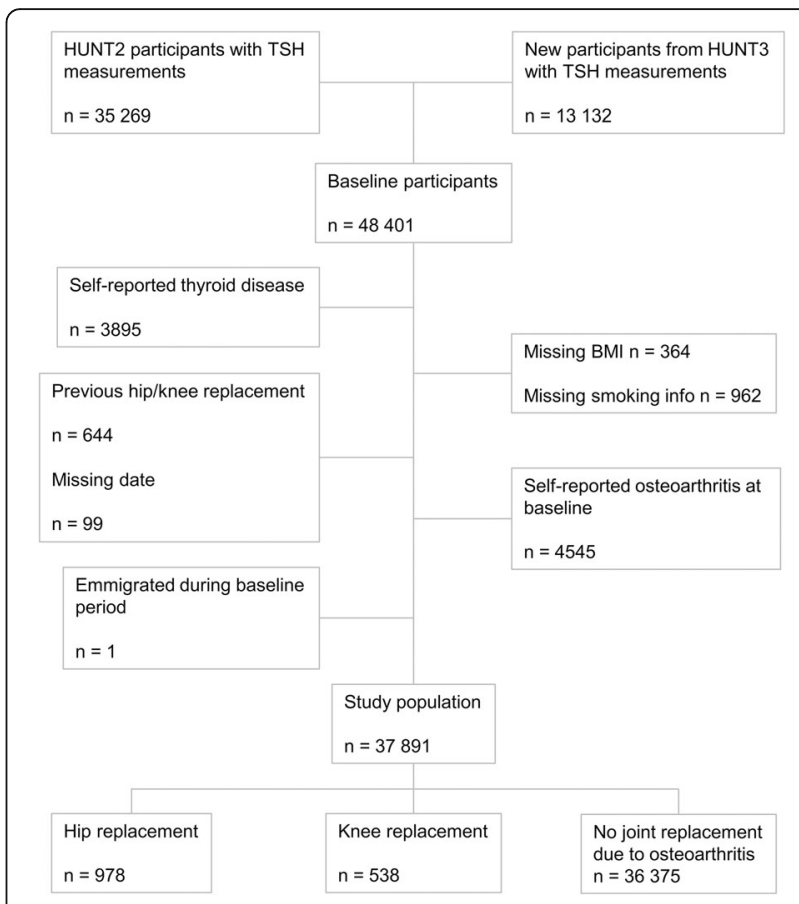

Fig. 1 Flowchart (TSH: Thyroid stimulating hormone)

BMI was calculated as weight in kilograms divided by squared height in metres.

A non-fasting venous blood sample was drawn from each participant. Concentrations of TSH, free thyroxine (fT4) and total triiodothyronine (T3) in HUNT2 were measured at the Hormone Laboratory, Aker University Hospital, Oslo, using DELFIA hTSH Ultra (sensitivity, $0.03 \mathrm{mU} / \mathrm{L}$; and total analytic variation $<5 \%$ ), DELFIA fT4 (total analytic variation $<7 \%$ ), and AutoDELFIA T3 (total analytic variation $<5 \%$ ), all from Wallac Oy, Turku, Finland. In HUNT3, serum TSH and fT4 were measured at Levanger Hospital, Levanger, Norway, using Architect cSystems ci8200 (sensitivity, $0.01 \mathrm{mU} / \mathrm{l}$; and a total analytic variation $<5 \%$ ), and Architect cSystems ci8200 (total analytic variation $<6 \%$ ), respectively, both from Abbott, Clinical Chemistry, USA. The measurement methods of TSH in HUNT2 and HUNT3 have previously been compared, with similar results [14], and agreement expressed by Bland-Altman [15] did not reveal any obvious pattern or deviations. The Norwegian population is considered to have sufficient iodine intake [16], and reference range for clinically normal TSH was defined as 0.50 to $3.5 \mathrm{mU} / \mathrm{l}$ based on previous publications from this population [13].

\section{Covariates}

The covariates were chosen based on previous literature, and had to be associated with both thyroid function and osteoarthritis. We mapped possible casual 
pathways between all variables using a directed acyclic graph model (DAG) (Appendix) to visually identify possible confounding pathways [17]. Based on this DAG-model, the possible confounders included in this analysis were sex, age (continuous), BMI (continuous), current smoking status (never, former or current), diabetes (yes or no) and physical activity. Physical activity was categorized by duration of light physical activity (none, $<1,1-2, \geq 3 \mathrm{~h} /$ week), and/or duration of hard physical activity (none, $<1,1-2, \geq 3 \mathrm{~h} /$ week). The physical activity questions have been previously validated among men, where especially hard physical activity correlated well with more objective measures [18]. These two variables were combined into one physical activity variable indicating intensity and duration: None (no activity), medium ( $\leq 2 \mathrm{~h} /$ week light physical activity and/ or $<1 \mathrm{~h}$ /week hard physical activity), hard ( $\geq 3 \mathrm{~h} /$ week light physical activity and/or $\geq 1 \mathrm{~h} /$ week hard physical activity).

Osteoarthritis at baseline was based on an affirmative answer to the question: "Has a doctor ever said that you have/have had osteoarthritis?" from HUNT2 participants, and "Have you had or do you have osteoarthritis?" from HUNT3 participants.

\section{Outcome}

In this study, primary total hip or knee replacement was considered to be a surrogate measure of severe osteoarthritis; and we used primary total hip or knee replacement due to primary osteoarthritis as outcome. The 11-digit identification numbers assigned to every Norwegian citizen enabled linkage to the Norwegian Arthroplasty Register (NAR). For each arthroplasty performed, the orthopaedic surgeon submits a standardized form containing information about the patient, the diagnosis that led to the arthroplasty, the procedure and the type of implant used [19]. NAR was established in 1987 and includes all artificial joints from 1994 onwards. The completeness of hip and knee replacement registration is over 95\% [20].

\section{Statistical methods}

The participants were placed in five categories according to their TSH level: One category indicating hyperthyroid function $(<0.50 \mathrm{mU} / \mathrm{l})$; three categories within the clinical reference range (0.50-1.49, $1.5-2.49$ and $2.5-3.5 \mathrm{mU} / \mathrm{l})$; and one category indicating hypothyroid function $(\geq 3.5$ $\mathrm{mU} / \mathrm{l}$ ) [21]. Hazard ratios (HRs) of THR or TKR by category of TSH were estimated using a Cox proportional hazards model with 95\% confidence interval (CI); TSH 1.5-2.49 mU/l was chosen as a reference. TSH was also analysed as both a continuous variable, and as logtransformed continuous TSH. The HRs were adjusted for age, sex, BMI and smoking. An additional analysis also adjusted for physical activity and diabetes. We treated THR and TKR both as separate outcomes and combined in one outcome variable of total joint replacement (TJR).

A sub-analysis included only persons that participated in both HUNT2 and HUNT3 $(n=19$ 397). TSH levels at HUNT3 were then subtracted from the TSH levels in HUNT2 to estimate the change in TSH during the intervening decade. Information on the other baseline covariates was taken from HUNT3. Among these participants a total of 7740 were excluded due to self-reported thyroid disease $(n=2744)$, missing information on BMI $(n=108)$, missing information on smoking $(n=621)$, previous THR or TKR $(n=928)$, missing date of operation $(n=29)$ or self-reported osteoarthritis at baseline $(n=3310)$. Thus, a total of 11657 people were eligible for this sub-analysis. This sub-population was also used in an analysis that investigated whether the results changed when those who had started on thyroid medication after baseline in HUNT2 were excluded.

In a sensitivity analysis people were divided into two groups, one with biochemically manifest (overt) hypothyroidism (defined as $\mathrm{TSH}>4.0 \mathrm{mU} / \mathrm{L}$ combined with $\mathrm{fT} 4<8.0 \mathrm{pmol} / \mathrm{L})$, and the other with overt hyperthyroidism (defined as $\mathrm{TSH}<0.10 \mathrm{mU} / \mathrm{L}$ and fT4 $>20.0 \mathrm{pmol} / \mathrm{L}$ and/or total T3 $>2.7 \mathrm{nmol} / \mathrm{L}$ ). This classification by overt hypo- or hyperthyroidism was made possible by the fT4 measurements taken in people whose TSH levels were $<0.20 \mathrm{mU} / \mathrm{l}$ or $>4.0$ $\mathrm{mU} / \mathrm{l}$ in HUNT2, and in people whose levels were $<0.10 \mathrm{mU} / 1$ or $>3.0 \mathrm{mU} / 1$ in HUNT3. Total T3 was only available in HUNT2 and only measured if TSH levels were $<0.20 \mathrm{mU} / \mathrm{L}$.

Two additional analyses were performed on the baseline population: First, we investigated the association between TSH level (categorical) and self-reported osteoarthritis at baseline by using a logistic regression model, adjusting for sex, age, BMI, smoking, physical activity and diabetes. Second, we compared the incidence rate of THR or TKR in participants with and without selfreported thyroid disease at baseline using Fisher's Exact test. After excluding participants with missing information on BMI and smoking, previous THR or TKR, missing date of operation or self-reported osteoarthritis at baseline, 2955 participants reported thyroid disease.

Proportional hazards assumptions were evaluated by Schoenfeld residuals tests. They showed proportional hazards on all covariates, except for age. Thus we did an additional stratified analysis on age, but it did not show different results (data not shown). Age was therefore kept as a continuous variable in all our analyses. All statistical analyses were two-sided with a significance level of $p<0.05$. The analyses were performed using Stata 14.0/SE (StataCorp LP, College Station, TX, USA). 


\section{Results}

Of the 37891 participants, 908 (2.4\%) had low TSH $(<0.50 \mathrm{mU} / \mathrm{l})$ indicating hyperthyroid function, and 2307 (6.1\%) had high TSH ( $\geq 3.6 \mathrm{mU} / \mathrm{l})$ indicating hypothyroid function (Table 1). Among the women, $6.9 \%$ had high TSH, compared to $4.8 \%$ of men. Participants with high TSH were generally older and were less likely to be current smokers than participants in the reference group (TSH 1.5-2.4 mU/l). No clear trend was seen in relation to physical activity.

In total, 978 received THR and 538 received TKR during a median follow-up time of 15.7 years (mean 12.3 years). At baseline, the mean age was 50.7 years (SD 15.8), and the mean ages at THR and TKR were 69.5 years (SD 8.9) and 69.4 years (SD 8.4), respectively.

TSH level did not influence the risk of THR or TKR in the unadjusted analysis or the analysis adjusted for gender, age, BMI and smoking (Table 2). Neither additional adjustment for physical activity and diabetes (Table 2), nor collapsing the outcome variable into total joint replacement (TJR) (Table 3), altered these results. Analyses using TSH as a continuous variable did not show any association between TSH and THR or TKR (Fig. 2). Additional log-transformation of TSH as a continuous variable did not significantly change these results (data not shown).

In a separate analysis studying the change in TSH (delta-TSH) during the time between HUNT2 and HUNT3, 11657 participants were included. Of these, 200 received THR and 102 received TKR during a mean follow-up time of 6.1 years. No association was seen between changes in TSH and risk of THR or TKR (Fig. 3). Additional adjustment for baseline TSH did not substantially alter these results. The same sub-population was also used to identify persons with no thyroid disease or treatment at baseline in HUNT2 who still had no thyroid disease or treatment at HUNT3. However, no association between TSH and THR (HR 1.00, 95\% CI $0.97-1.02$ ) or TKR (HR $0.98,95 \%$ CI $0.94-1.03$ ) was found.

In a sensitivity analysis neither overt hypo- nor hyperthyroid function was found to influence the risk of THR or TKR, (HR 0.91, 95\% CI 0.40-2.04) and (HR 2.01, 95\% CI 0.75-5.4) respectively. Also, no association was found between overt hypothyroid function and TKR (HR 1.03, 95\% CI 0.38-2.78). There were no cases of TKR amongst the overtly hyperthyroid, so no analysis could be performed in this subgroup.

In additional analyses on the baseline population, there was no association between TSH levels and self-reported osteoarthritis at baseline (data not shown). Those with self-reported thyroid disease at baseline had an incidence rate of 0.0027 THR per person-year and 0.0015 TKR per person-year. Our main study population (excluding those with self-reported thyroid disease) had an incidence rate of 0.0021 THR per person-year and 0.0012 TKR per person-year. This gave and incidence rate ratio of 1.28 (95\% CI 1.04-1.56) for THR, and 1.27 (95\% CI 0.95-1.95) for TKR, and indicated a slightly higher incidence rate for THR in those with self-reported thyroid disease, but no significant difference in the incidence rate for TKR, compared to participants without self-reported thyroid disease.

\section{Discussion}

In this large prospective study we did not find any association between thyroid function and the risk of THR or

Table 1 Study population characteristics in relation to thyroid stimulating hormone (TSH) categories

\begin{tabular}{|c|c|c|c|c|c|c|c|c|c|c|c|c|}
\hline \multirow[b]{3}{*}{ Participants (\%) } & \multirow{2}{*}{\multicolumn{2}{|c|}{$<0.50$}} & \multicolumn{4}{|c|}{ Serum TSH } & \multirow{2}{*}{\multicolumn{2}{|c|}{$2.5-3.49$}} & \multirow{2}{*}{\multicolumn{2}{|c|}{$\geq 3.5$}} & \multicolumn{2}{|l|}{ Total } \\
\hline & & & \multicolumn{2}{|c|}{$0.50-1.49$} & \multicolumn{2}{|c|}{$1.5-2.49$} & & & & & & \\
\hline & 908 & $(2.4)$ & 17675 & $(46.7)$ & 13228 & (34.9) & 3773 & $(10.0)$ & 2307 & $(6.1)$ & 37891 & \\
\hline Women (\%) & 651 & $(2.9)$ & 10405 & $(45.8)$ & 7738 & $(34.1)$ & 2346 & $(10.3)$ & 1574 & $(6.9)$ & 22714 & $(60.0)$ \\
\hline Men (\%) & 257 & $(1.7)$ & 7270 & $(47.9)$ & 5490 & $(36.2)$ & 1427 & $(9.4)$ & 733 & $(4.8)$ & 15177 & $(40.0)$ \\
\hline Age (SD) & 50.7 & $(16.7)$ & 48.2 & (15.3) & 51.6 & (15.6) & 54.9 & $(15.9)$ & 58.3 & (15.3) & 50.7 & $(15.8)$ \\
\hline BMI (SD) & 25.9 & $(4.4)$ & 26.1 & $(4.1)$ & 26.8 & $(4.2)$ & 27.1 & $(4.4)$ & 27.2 & $(4.5)$ & 26.5 & $(4.2)$ \\
\hline \multicolumn{13}{|l|}{ Smoking status (\%) } \\
\hline Never & 335 & $(36.9)$ & 6778 & (38.3) & 6080 & $(46.0)$ & 1869 & $(49.5)$ & 1167 & $(50.6)$ & 16229 & $(42.8)$ \\
\hline Former & 236 & $(26.0)$ & 4683 & $(26.5)$ & 3768 & $(28.5)$ & 1120 & $(29.7)$ & 723 & (31.3) & 10530 & $(27.8)$ \\
\hline Current & 337 & $(37.1)$ & 6214 & $(35.2)$ & 3380 & $(25.5)$ & 784 & $(20.8)$ & 417 & $(18.1)$ & 11132 & $(29.4)$ \\
\hline \multicolumn{13}{|c|}{ Physical activity (\%) (missing = 6826) } \\
\hline Low & 46 & $(6.5)$ & 862 & $(6.0)$ & 719 & $(6.6)$ & 232 & $(7.4)$ & 151 & $(8.2)$ & 2010 & $(6.5)$ \\
\hline Medium & 371 & $(52.8)$ & 7226 & $(49.9)$ & 5454 & $(50.0)$ & 1555 & $(49.7)$ & 921 & $(50.1)$ & 15527 & $(50.0)$ \\
\hline High & 286 & $(40.7)$ & 6394 & $(44.1)$ & 4738 & $(43.4)$ & 1344 & $(42.9)$ & 766 & $(41.7)$ & 13528 & (43.6) \\
\hline Diabetes $(\%)($ missing $=56)$ & 55 & $(6.1)$ & 613 & $(3.5)$ & 559 & $(4.2)$ & 173 & $(4.6)$ & 115 & $(5.0)$ & 1515 & 4.0 \\
\hline
\end{tabular}


Table 2 Association between TSH categories and hip or knee replacement due to osteoarthritis

\begin{tabular}{|c|c|c|c|c|c|c|c|c|}
\hline TSH (mU/L) & Persons (n) & Cases (n) & $\mathrm{HR}^{\mathrm{a}}$ & $(95 \% \mathrm{Cl})$ & $\mathrm{HR}^{\mathrm{b}}$ & $(95 \%$ Cl) & $H R^{c}$ & $(95 \% \mathrm{Cl})$ \\
\hline \multicolumn{9}{|l|}{ THR } \\
\hline$<0.50$ & 908 & 21 & 0.98 & $(0.63-1.53)$ & 0.99 & $(0.64-1.53)$ & 1.04 & $(0.63-1.69)$ \\
\hline $0.50-1.49$ & 17675 & 411 & 0.93 & $(0.81-1.08)$ & 1.07 & $(0.93-1.24)$ & 1.09 & $(0.94-1.28)$ \\
\hline $1.5-2.49$ & 13228 & 359 & 1 & Reference & 1 & Reference & 1 & Reference \\
\hline $2.5-3.49$ & 3773 & 105 & 0.98 & $(0.79-1.22)$ & 0.85 & $(0.68-1.05)$ & 0.88 & $(0.69-1.11)$ \\
\hline$>3.5$ & 2307 & 82 & 1.22 & $(0.96-1.55)$ & 0.96 & $(0.76-1.22)$ & 0.98 & $(0.75-1.27)$ \\
\hline \multicolumn{9}{|l|}{ TKR } \\
\hline$<0.50$ & 908 & 14 & 1.26 & $(0.73-2.16)$ & 1.32 & $(0.76-2.26)$ & 1.33 & $(0.74-2.4)$ \\
\hline $0.50-1.49$ & 17675 & 224 & 0.97 & $(0.80-1.18)$ & 1.15 & $(0.95-1.40)$ & 1.12 & $(0.91-1.37)$ \\
\hline $1.5-2.49$ & 13228 & 191 & 1 & Reference & 1 & Reference & 1 & Reference \\
\hline $2.5-3.49$ & 3773 & 64 & 1.12 & $(0.84-1.48)$ & 0.94 & $(0.71-1.25)$ & 0.88 & $(0.65-1.20)$ \\
\hline$>3.5$ & 2307 & 45 & 1.24 & $(0.90-1.72)$ & 0.98 & $(0.70-1.35)$ & 0.87 & $(0.61-1.25)$ \\
\hline
\end{tabular}

(THR total hip replacement, TKR total knee replacement)

anadjusted

${ }^{\mathrm{b}}$ Adjusted for age, sex, BMI and smoking

${ }^{\mathrm{C}}$ Adjusted for age, sex, BMI, smoking, physical activity and diabetes

TKR due to osteoarthritis. Neither were changes in TSH over time, or overt hypo- or hyperthyroidism, associated with incidence of THR or TKR.

Few previous population studies have investigated the association of thyroid function with risk of osteoarthritis. In 1996, a cross-sectional study of 577 men and 798 women found no evidence of a significant association between current thyroid status and either chondrocalcinosis or osteoarthritis [10]. However, that study only investigated prevalent osteoarthritis with a concurrent serum TSH concentration and could not take into account development in TSH or later treatment for abnormal thyroid function. Since our study could use data from both the second and third waves of the HUNTsurvey, we were able to investigate the development of TSH over a median time of 11.2 years (SD 0.6). Change in TSH over time was however not associated with osteoarthritis development resulting in the need for joint replacement.

Previous or current thyroid disease at baseline was an exclusion criterion in our study. Nonetheless, all participants with TSH levels suggesting hypothyroid or hyperthyroid function may have received medical treatment for thyroid disease during the follow-up period, as participants with biochemical indication of pathological thyroid function were recommended to contact their general practitioners [22]. This could have weakened any association between TSH and osteoarthritis. We therefore did a sub-analysis of persons that participated in both HUNT2 and HUNT3, and excluded participants that reported use of thyroid medication or thyroid disease in HUNT3. This did not significantly alter the results.

Our findings must be interpreted in relation to recent genetic studies on intracellular T3 availability in joint cartilage. There has been an increased interest in the effect of deiodinase polymorphisms on osteoarthritis [5]. Iodothyronine deiodinases represent a family of proteins involved in local homeostasis of thyroxine (T4) and triiodothyronine (T3). Three deiodinases have been described and, of these, the deiodinase type 2 (D2) and deiodinase type 3 (D3) are

Table 3 Association between TSH categories and total joint replacement (TJR) in the hip or knee due to osteoarthritis

\begin{tabular}{lllllllll}
\hline TSH $(\mathrm{mU} / \mathrm{L})$ & Persons $(\mathrm{n})$ & Cases $(\mathrm{n})$ & $\mathrm{HR}^{\mathrm{a}}$ & $(95 \% \mathrm{Cl})$ & $\mathrm{HR}^{\mathrm{b}}$ & $(95 \% \mathrm{Cl})$ & $\mathrm{HR}^{\mathrm{C}}$ & $(95 \% \mathrm{Cl})$ \\
\hline$<0.50$ & 908 & 35 & 1.08 & $(0.77-1.52)$ & 1.10 & $(0.78-1.55)$ & 1.15 & $(0.79-1.67)$ \\
$0.50-1.49$ & 17675 & 635 & 0.95 & $(0.84-1.06)$ & 1.10 & $(0.98-1.23)$ & 1.10 & $(0.97-1.25)$ \\
$1.5-2.49$ & 13228 & 550 & 1 & Reference & 1 & Reference & 1 & Reference \\
$2.5-3.49$ & 3773 & 169 & 1.03 & $(0.87-1.22)$ & 0.88 & $(0.74-1.05)$ & 0.88 & $(0.73-1.06)$ \\
$>3.5$ & 2307 & 127 & 1.23 & $(1.01-1.49)$ & 0.97 & $(0.80-1.17)$ & 0.94 & $(0.76-1.16)$ \\
\hline
\end{tabular}

anadjusted

${ }^{\mathrm{b}}$ Adjusted for age, sex, BMI and smoking

${ }^{\mathrm{C}}$ Adjusted for age, sex, BMI, smoking, physical activity and diabetes 


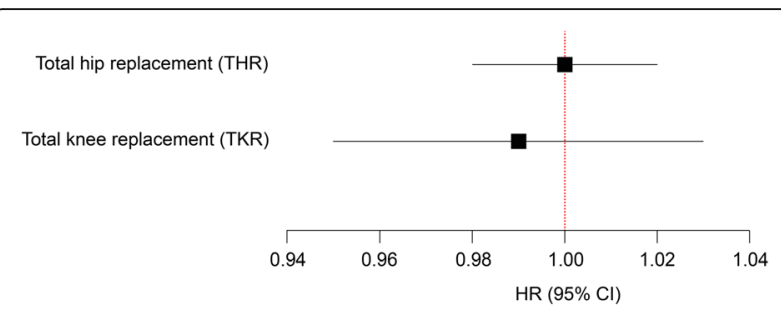

Fig. 2 Association between continuous TSH and total hip replacement (THR) or total knee replacement (TKR) due to osteoarthritis. (Adjusted for age, sex, BMl, smoking, physical activity and diabetes)

detected in bone and cartilage. D2 plays a major role in conversion of T4 to biologically active T3 [23] and thus upregulates local T3 levels. Deiodinase type 3 (D3) is the main T3-inactivating enzyme and consequently downregulates the local T3 levels. T3 is considered an important regulator of chondrocyte cell growth and differentiation in the endochondral growth plate [24]. Local T3 availability, regulated by the opposite functions of D2 and D3, may be a determinant of osteoarthritis development. D2 has been reported to be upregulated in the cartilage of joints affected by osteoarthritis compared to joints unaffected by osteoarthritis [25, 26]. However, it is not known if this is a result of the ongoing osteoarthritis process, or a reflection of the underlying disease pathway. Taken together, these findings suggest that deiodinase regulated local availability of $\mathrm{T} 3$ in chondrocytes is a possible factor in the pathophysiology of osteoarthritis [27, 28]. Since our study did not find any association between circulating $\mathrm{TSH}, \mathrm{T} 3 / \mathrm{T} 4$ levels and osteoarthritis, it is conceivable that the serum thyroid hormone levels may be independent of local intracellular T3 levels in joints. Another possible explanation could be that polymorphism in the gene coding for D2 creates a predisposition for non-optimal bone shape $[29,30]$, leading to increased risk of osteoarthritis independent of local thyroid hormone levels.

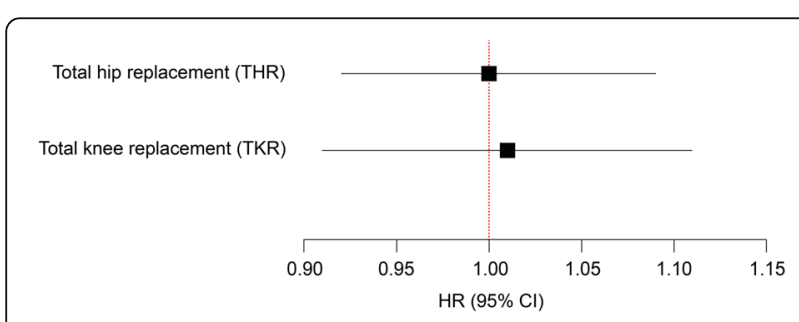

Fig. 3 Association between change in TSH (delta TSH) and total hip replacement (THR) or total knee replacement (TKR) due to osteoarthritis. (Adjusted for age, sex, BMl and smoking)

\section{Strengths and limitations}

Our study included over 37000 persons without known thyroid disease at baseline, and in most cases thyroid function was measured many years prior to joint replacement. To the best of our knowledge, this is the first prospective population study addressing the association between thyroid function and joint replacement due to primary osteoarthritis. The prospective design and longitudinal data on TSH measurements are strengths of this study. By excluding participants with self-reported osteoarthritis at baseline it was possible to differentiate between risk of osteoarthritis development and progression.

Our study used joint replacement due to primary osteoarthritis as a surrogate measure of severe osteoarthritis. Validation of the osteoarthrosis diagnosis from the Norwegian Arthroplasty Register has not been done in an unselected population [31]. However, the Danish hip Arthroplasty Registry has reported a positive predictive value of $85 \%$ regarding primary hip osteoarthritis diagnosis [32], and it is likely that these results are comparable to the Norwegian Arthroplasty Register. The advantage of using joint replacement as a proxy for osteoarthritis is its unambiguous connection with disease burden of osteoarthritis compared to other osteoarthritis definitions, e.g., radiographic criteria, symptom criteria or osteoarthritis defined by self-reported diagnosis [33]. However, this outcome measure is still limited in some respects, most importantly, that patients' health status and potential comorbidities influence orthopaedic surgeons' choices regarding operative treatment. Secondly, persons with moderate osteoarthritis who engage in demanding physical activities could be more motivated to have surgery than less active persons. Persons who are generally inactive may be less motivated to have surgery even if they have more severe osteoarthritis. This could give a healthy patient selection bias with corresponding underestimation of the effect of thyroid function.

Previous injuries increase the risk of knee osteoarthritis [34, 35], but only joint replacements due to primary/idiopathic osteoarthritis were included in our study. We did not have direct information on previous injury, but we excluded all cases in which the operating surgeon reported that the knee joint replacement was due to sequela from fracture, ligament injury, meniscal injury, infection, rheumatoid arthritis or ankylosing spondylitis.

The interrelationship between BMI and thyroid function is complex and BMI could be treated as either a confounder or a mediator in our model [36]. The reason we chose to define it as a confounder was that we wanted to investigate the direct effect of thyroid function on joint replacement, independent of BMI. Potential 
confounding by other unmeasured factors could not be excluded. But these factors should then be associated with both thyrotropin level and osteoarthritis. Therefore, we did not adjust for level of education in this study (Appendix).

The participation rate in the HUNT-surveys was fairly high compared to most other surveys, but there is always a potential risk of selection bias that cannot be adjusted for in the statistical analysis [37]. Blood samples were not drawn at a set time of the day, and it is known that other factors like exercise and sleep deprivation influence TSH levels [38]. This might have led to nondifferential classification bias, thus weakening any associations. We also only had data on $\mathrm{fT} 4$ and T3 in subpopulations. Therefore, the absolute numbers of participants with overt hypo- or hyperthyroidism were small, reducing the power to detect any association between them and euthyroid subject and should thus be interpreted with caution.

This study focused on the relationship between thyroid function and osteoarthritis. However, there may also be an association between autoimmune thyroid disease and osteoarthritis [39]. As thyroid autoantibody not necessarily correlate with thyroid function, an association between thyroid function and osteoarthritis trough autoimmune factors could be missed in our study.

The exclusion of participants who reported hypo- or hyperthyroidism in their answers regarding use of treatment or medication might have caused misclassification since radioiodine is used in the treatment of cancer and T4 in the medical treatment of goitre. However, these treatments are infrequent, and it is unlikely that they substantially altered our results.

In the analysis comparing incidence rate of THR in people with and without self-reported thyroid disease we found a small, but significant, increased risk of THR in people reporting thyroid disease. And since we excluded participants with self-reported thyroid disease, this might have led to an underestimation of the effect on THR. We therefore did an additional analysis including those with self-reported thyroid disease: This showed no association between TSH levels and THR in a Cox-regression model (data not shown), and thus confirmed the findings from the primary analysis.

\section{Conclusion}

In this prospective study of 37891 participants without previously known thyroid disease, we did not find that thyroid function was associated with risk of hip or knee joint replacement due to osteoarthritis. Neither did we find any association between TSH development over time and risk of hip or knee joint replacement due to osteoarthritis.

\section{Appendix}

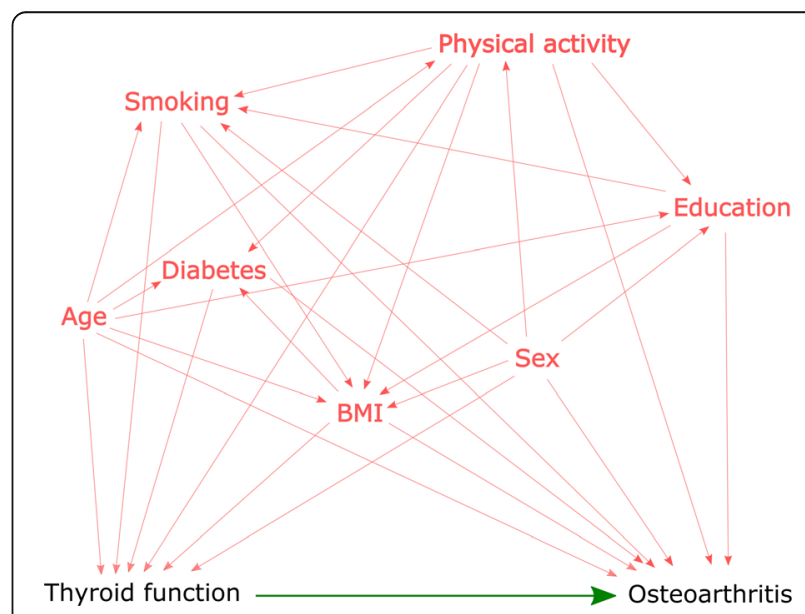

Fig. 4 Direct Acyclic Graph (DAG) - analysis. Figure showing possible casual pathways between variables

\section{Abbreviations}

BMl: Body mass index; D2: Deiodinase type 2; D3: Deiodinase type 3; DAG: Directed acyclic graph; fT4: Free thyroxine; HR: Hazard ratio; HUNT: The Nord-Trøndelag Health study; NAR: Norwegian Arthroplasty Register; OA: Osteoarthritis; SD: Standard deviation; T3: Triiodothyronine; T4: Thyroxine; THR: Total hip replacement; TKR: Total knee replacement; TSH: Thyroid stimulating hormone

\section{Acknowledgements}

The Nord-Trøndelag Health Study is a collaborative effort of the Faculty of Medicine, Norwegian University of Science and Technology; the Norwegian Institute of Public Health; and the Nord-Trøndelag County Council. The Norwegian Arthroplasty Register is owned by the Norwegian Orthopedic Association and administered by the Orthopedic Department at Haukeland University Hospital, Bergen, Norway.

\section{Funding}

This study was supported by research grants from the Liaison Committee between the Central Norway Regional Health Authority (RHA) and the Norwegian University of Science and Technology (NTNU), the Dr. Egil Kjeldaas foundation (Lege Egil Kjeldaas legat), the Norwegian Orthopaedic Association and Levanger Hospital, Nord-Trøndelag Hospital Trust. The funding sources had no involvement in the study design, the collection, analysis or interpretation of data, writing the paper or the decision to submit the paper for publication.

\section{Availability of data and materials}

Ethical and legal restrictions prohibit the authors from making datasets available outside the HUNT database, which is available by contacting the HUNT administration. Please see https://www.ntnu.edu/hunt/data for details on how to obtain all relevant data.

\section{Authors' contributions}

AL conceived of the idea for this study. AlH participated in the study concept and design, obtained funding, performed the analysis, interpreted the data and drafted the manuscript. LN, MBJ, AL, GBF, OF, KS and JAZ were involved in the conception and design of the study. OF was also involved in the collection of hip and knee replacement data. AMF contributed statistical expertise. All the authors revised the manuscript for important intellectual content and approved the final version of the manuscript.

\section{Competing interests}

The authors declare that they have no competing interests. 


\section{Consent for publication}

Not applicable.

\section{Ethics approval and consent to participate}

The participants signed written informed consent for participation in HUNT, NAR and linkage of data to national health registries. This study was approved by the Norwegian Regional Committee for Ethics in medical Research (2013/ 151/REK Sør-Øst C)

\section{Publisher's Note}

Springer Nature remains neutral with regard to jurisdictional claims in published maps and institutional affiliations.

\section{Author details}

${ }^{1}$ The HUNT Research Centre, Department of Public Health and Nursing, Faculty of Medicine and Health Sciences, Norwegian University of Science and Technology (NTNU), Levanger, Norway. ${ }^{2}$ Division of Orthopaedic Surgery, Oslo University Hospital, Oslo, Norway. ${ }^{3}$ Research and Communication Unit for Musculoskeletal Health, Division of Clinical Neuroscience, Oslo University Hospital, Oslo, Norway. ${ }^{4}$ The Norwegian Arthroplasty Register, Department of Orthopedic Surgery, Haukeland University Hospital, Bergen, Norway. ${ }^{5}$ Department of Clinical Medicine, Institute of Medicine and Dentistry, University of Bergen, Bergen, Norway. ${ }^{6}$ Faculty of Medicine, University of Oslo, Oslo, Norway.

Received: 20 December 2016 Accepted: 9 May 2017 Published online: 18 May 2017

\section{References}

1. Vos T, Flaxman AD, Naghavi M, Lozano R, Michaud C, Ezzati M, Shibuya K, Salomon JA, Abdalla S, Aboyans V, et al. Years lived with disability (YLDs) for 1160 sequelae of 289 diseases and injuries 1990-2010: a systematic analysis for the Global Burden of Disease Study 2010. Lancet. 2012;380(9859):2163-96.

2. Goldring MB, Berenbaum F. Emerging targets in osteoarthritis therapy. Curr Opin Pharmacol. 2015;22:51-63.

3. Svege I, Nordsletten L, Fernandes L, Risberg MA. Exercise therapy may postpone total hip replacement surgery in patients with hip osteoarthritis: a long-term follow-up of a randomised trial. Ann Rheum Dis. 2015;74(1):164-9.

4. Williams GR. Thyroid Hormone Actions in Cartilage and Bone. European Thyroid J. 2013;2(1):3-13.

5. Verloop H, Dekkers OM, Peeters RP, Schoones JW, Smit JW. Genetics in endocrinology: genetic variation in deiodinases: a systematic review of potential clinical effects in humans. Eur J Endocrinol. 2014;171(3):R123-135.

6. Waung JA, Bassett JH, Williams GR. Adult mice lacking the type 2 iodothyronine deiodinase have increased subchondral bone but normal articular cartilage. Thyroid. 2015;25(3):269-77.

7. Ladenson PW, Kristensen JD, Ridgway EC, Olsson AG, Carlsson B, Klein I, Baxter JD, Angelin B. Use of the thyroid hormone analogue eprotirome in statin-treated dyslipidemia. N Engl J Med. 2010;362(10):906-16.

8. Sjouke B, Langslet G, Ceska R, Nicholls SJ, Nissen SE, Ohlander M, Ladenson PW, Olsson AG, Hovingh GK, Kastelein JJ. Eprotirome in patients with familial hypercholesterolaemia (the AKKA trial): a randomised, double-blind, placebocontrolled phase 3 study. Lancet Diabetes Endocrinol. 2014;2(6):455-63.

9. Lammel Lindemann J, Webb P. Sobetirome: the past, present and questions about the future. Expert Opin Ther Targets. 2016;20(2):145-9.

10. Chaisson CE, McAlindon TE, Felson DT, Naimark A, Wilson PW, Sawin CT. Lack of association between thyroid status and chondrocalcinosis or osteoarthritis: the Framingham Osteoarthritis Study. J Rheumatol. 1996;23(4):711-5.

11. HUNT home page [http://www.ntnu.no/hunt]. Accessed 15 Nov 2016

12. Krokstad S, Langhammer A, Hveem K, Holmen TL, Midthjell K, Stene TR, Bratberg G, Heggland J, Holmen J. Cohort Profile: the HUNT Study, Norway. Int J Epidemiol. 2013;42(4):968-77.

13. Bjoro T, Holmen J, Kruger O, Midthjell K, Hunstad K, Schreiner T, Sandnes L, Brochmann $\mathrm{H}$. Prevalence of thyroid disease, thyroid dysfunction and thyroid peroxidase antibodies in a large, unselected population. The Health Study of Nord-Trondelag (HUNT). Eur J Endocrinol. 2000;143(5):639-47.

14. Svare A, Nilsen TI, Bjoro T, Asvold BO, Langhammer A. Serum TSH related to measures of body mass: longitudinal data from the HUNT Study, Norway. Clin Endocrinol (Oxf). 2011;74(6):769-75.

15. Bland JM, Altman DG. Statistical methods for assessing agreement between two methods of clinical measurement. Lancet. 1986;1(8476):307-10.
16. Kapelrud $H$, Frey $H$, Theodorsen L. [Excretion of iodine in the urine. A study from 6 different Norwegian districts in 1985]. Tidsskr Nor Laegeforen. 1987; 107(15):1320-1. 1317.

17. Suttorp MM, Siegerink B, Jager KJ, Zoccali C, Dekker FW. Graphical presentation of confounding in directed acyclic graphs. Nephrol Dial Transplant. 2015;30(9):1418-23.

18. Kurtze N, Rangul V, Hustvedt B-E, Flanders WD. Reliability and validity of selfreported physical activity in the Nord-Trøndelag Health Study (HUNT 2). Eur J Epidemiol. 2007;22(6):379-87.

19. Havelin LI, Engesaeter LB, Espehaug B, Furnes O, Lie SA, Vollset SE. The Norwegian Arthroplasty Register: 11 years and 73,000 arthroplasties. Acta Orthop Scand. 2000;71(4):337-53.

20. Espehaug B, Furnes O, Havelin LI, Engesaeter LB, Vollset SE, Kindseth O. Registration completeness in the Norwegian Arthroplasty Register. Acta Orthop. 2006;77(1):49-56.

21. Hellevik Al, Asvold BO, Bjoro T, Romundstad PR, Nilsen TI, Vatten LJ. Thyroid function and cancer risk: a prospective population study. Cancer Epidemiol Biomarkers Prev. 2009;18(2):570-4.

22. HUNT databank [ https://hunt-db.medisin.ntnu.no/hunt-db/ ]. Accessed 15 Nov 2016.

23. Kohrle J. Local activation and inactivation of thyroid hormones: the deiodinase family. Mol Cell Endocrinol. 1999;151(1-2):103-19.

24. Robson H, Siebler T, Stevens DA, Shalet SM, Williams GR. Thyroid hormone acts directly on growth plate chondrocytes to promote hypertrophic differentiation and inhibit clonal expansion and cell proliferation. Endocrinology. 2000;141(10):3887-97.

25. Bos SD, Bovee JV, Duijnisveld BJ, Raine EV, van Dalen WJ, Ramos YF, van der Breggen R, Nelissen RG, Slagboom PE, Loughlin J, et al. Increased type II deiodinase protein in OA-affected cartilage and allelic imbalance of OA risk polymorphism rs225014 at DIO2 in human OA joint tissues. Ann Rheum Dis. 2012;71(7):1254-8

26. Meulenbelt I, Bos SD, Chapman K, van der Breggen R, Houwing-Duistermaat J, Kremer D, Kloppenburg M, Carr A, Tsezou A, Gonzalez A, et al. Metaanalyses of genes modulating intracellular T3 bio-availability reveal a possible role for the DIO3 gene in osteoarthritis susceptibility. Ann Rheum Dis. 2011;70(1):164-7.

27. Waung JA, Bassett JH, Williams GR. Thyroid hormone metabolism in skeletal development and adult bone maintenance. Trends Endocrinol Metab. 2012; 23(4):155-62.

28. Bay-Jensen AC, Slagboom E, Chen-An P, Alexandersen P, Qvist P Christiansen C, Meulenbelt I, Karsdal MA. Role of hormones in cartilage and joint metabolism: understanding an unhealthy metabolic phenotype in osteoarthritis. Menopause. 2013;20(5):578-86.

29. Waarsing $\mathrm{JH}$, Kloppenburg M, Slagboom PE, Kroon HM, HouwingDuistermaat JJ, Weinans H, Meulenbelt I. Osteoarthritis susceptibility genes influence the association between hip morphology and osteoarthritis. Arthritis Rheum. 2011:63(5):1349-54.

30. Meulenbelt I, Min JL, Bos S, Riyazi N, Houwing-Duistermaat JJ, van der Wijk HJ, Kroon HM, Nakajima M, Ikegawa S, Uitterlinden AG, et al. Identification of DIO2 as a new susceptibility locus for symptomatic osteoarthritis. Hum Mol Genet. 2008;17(12):1867-75.

31. Engesaeter IO, Lehmann T, Laborie LB, Lie SA, Rosendahl K, Engesaeter LB. Total hip replacement in young adults with hip dysplasia: age at diagnosis, previous treatment, quality of life, and validation of diagnoses reported to the Norwegian Arthroplasty Register between 1987 and 2007. Acta Orthop. 2011;82(2):149-54

32. Pedersen A, Johnsen S, Overgaard S, Soballe K, Sorensen HT, Lucht U. Registration in the danish hip arthroplasty registry: completeness of total hip arthroplasties and positive predictive value of registered diagnosis and postoperative complications. Acta Orthop Scand. 2004;75(4):434-41.

33. Kim C, Nevitt MC, Niu J, Clancy MM, Lane NE, Link TM, Vlad S, Tolstykh I, Jungmann PM, Felson DT, et al. Association of hip pain with radiographic evidence of hip osteoarthritis: diagnostic test study. BMJ. 2015;351:h5983.

34. Oiestad BE, Holm I, Engebretsen L, Aune AK, Gunderson R, Risberg MA. The prevalence of patellofemoral osteoarthritis 12 years after anterior cruciate ligament reconstruction. Knee Surg Sports Traumatol Arthrosc. 2013;21(4):942-9.

35. Murphy L, Schwartz TA, Helmick CG, Renner JB, Tudor G, Koch G, Dragomir A, Kalsbeek WD, Luta G, Jordan JM. Lifetime risk of symptomatic knee osteoarthritis. Arthritis Rheum. 2008;59(9):1207-13.

36. Pearce EN. Thyroid hormone and obesity. Curr Opin Endocrinol Diabetes Obes. 2012;19(5):408-13. 
37. Holmen J, Midthjell K, Krüger $\mathrm{O}$, Langhammer $\mathrm{A}$, Holmen $T L$, Bratberg G, Vatten LG. L-LP: The Nord-Trøndelag Health Study 1995-97 (HUNT 2): Objectives, contents, methods and participation. Norsk Epidemiologi. 2003;13(1):19-32.

38. Surks Ml, Goswami G, Daniels GH. The thyrotropin reference range should remain unchanged. J Clin Endocrinol Metab. 2005;90(9):5489-96.

39. Soy M, Guldiken S, Arikan E, Altun BU, Tugrul A. Frequency of rheumatic diseases in patients with autoimmune thyroid disease. Rheumatol Int. 2007; 27(6):575-7.

Submit your next manuscript to BioMed Central and we will help you at every step:

- We accept pre-submission inquiries

- Our selector tool helps you to find the most relevant journal

- We provide round the clock customer support

- Convenient online submission

- Thorough peer review

- Inclusion in PubMed and all major indexing services

- Maximum visibility for your research

Submit your manuscript at www.biomedcentral.com/submit 